\title{
A 7-YEAR-OLD BOY WITH HAND TREMORS AND A NOVEL MUTATION FOR L-2-HYDROXYGLUTARIC ACIDURIA
}

\author{
Olgac $\mathrm{A}^{1, *}$, Tekin Orgun L ${ }^{2}$, Ezgü FS ${ }^{1}$, Biberoğlu $\mathrm{G}^{1}$, Tümer $\mathrm{L}^{1}$ \\ *Corresponding Author: Dr. Asburce Olgac, Department of Pediatric Metabolism and Nutrition, Gazi \\ University Hospital, Mevlana Bulvar1, Ankara, Turkey. Tel: +90-533-962-7800. Fax: +90-312-202-6027. \\ E-mail: mabolgac@yahoo.com
}

\begin{abstract}
L-2-hydroxyglutaric aciduria (L2HGA), which is a rare autosomal recessive metabolic disorder caused by mutations in the encoding $\mathrm{L} 2 \mathrm{HGDH}$ gene. Neurological symptoms are the main predominant clinical signs. The distinctive feature is the specific multifocal lesion of the white matter detected on magnetic resonance imaging (MRI). A 7-year-old male patient of Turkish origin was admitted to the hospital because of hand tremors. Physical examination revealed macrocephaly, intention tremors, walking disability and ataxic gait. Urine organic acid analysis showed increased excretion of L-2-hydroxyglutaric acid (L2HG acid). Analysis of the $\mathrm{L} 2 \mathrm{HGDH}$ gene revealed a novel homozygous c.368A>G, p. (Tyr123Cys) mutation. L-2-hydroxyglutaric aciduria is a cerebral organic aciduria that may lead to various neurological complications. Early recognition of symptoms of L2HGA is important for initiation of supportive therapy that may slow down the progression of the disease.
\end{abstract}

Keywords: Inborn errors of metabolism; L-2-hydroxyglutaric aciduria (L2HGA); Seizure; Tremor.

\section{INTRODUCTION}

L-2-Hydroxyglutaric aciduria (L2HGA) is a rare cerebral organic aciduria occurring due to defects in L-2-hydroxyglutarate dehydrogenase (L2HGDH), encoded by the $L 2 H G D H$ gene, a mitochondrial enzyme that catalyses

\footnotetext{
${ }^{1}$ Department of Pediatric Metabolism and Nutrition, Gazi University Hospital, Ankara, Turkey

2 Department of Pediatric Neurology, Gazi University Hospital, Ankara, Turkey
}

the oxidation of L-2-hydroxyglutaric acid (L2HG acid) to $\alpha$-ketoglutarate [1-3]. The dysfunction of this enzyme leads to accumulation of $\mathrm{L} 2 \mathrm{HG}$ in urine, cerebrospinal fluid and plasma, which is the biochemical hallmark. The disease has a slowly progressive course and clinical signs are predominantly neurological symptoms, rarely leading to early loss of gross motor skills [1-3]. We present the case of a 7-year-old boy with L2HGA, with a novel mutation in the $L 2 H G D H$ gene.

\section{CASE REPORT}

A 7-year-old male patient of Turkish origin, was admitted to the hospital because of hand tremors. He was the first child of consanguineous parents (first degree cousins), with an uncomplicated prenatal and natal history, and slight delay of motor milestones. His hand tremors were noticed by his teacher during the first days of elementary school because of difficulty in writing. Written informed consent was obtained from the parents of the patient for DNA analysis, and publication of this case report and any accompanying images.

Upon admission, physical examination revealed macrocephaly (head circumference: $54 \mathrm{~cm}, 97$ th percentile), with normal weight and height percentiles (weight: $22 \mathrm{~kg}$, 25th-50th percentile, height: $124 \mathrm{~cm}, 50$ th percentile). He had intention tremors, walking disability and a slightly ataxic gait. Deep tendon reflexes of lower extremities were hyperactive and clonus was present. Psychometric evaluation showed delayed progression of language (age 3), social (age 5) and fine motor skills (age 5).

Laboratory analysis showed a normal complete blood count, biocehmistry, blood gas analysis, renal and liver function tests and electrolyte levels. Thyroid function tests, vitamin B12 and folic acid levels, autoimmune markers 
markers including ANA, anti-ds DNA, C-ANCA, P-AN$\mathrm{CA}$, were within reference ranges.

Cranial magnetic resonance imaging (MRI) showed signal changes in the supratentorial region, subcortical white matter, caudate and lentiform nuclei with sparing of the peri ventricular white matter, without any sign of cerebellar involvement [Figure 1(a), 1(b), 1(c)]. In accordance with the cranial MRI findings, an inborn error of metabolism was suspected, and metabolic tests were performed. may reveal increased 2-hydroclutaric acid, but the chiral configuration ( $\mathrm{D}$ or $\mathrm{L}$ form) has to be determined by GC-MS or liquid chromatography-tandem mass spectrometry (LC-MS/MS), as D-2-hydroxyglutaric aciduria (D2HGA) and L2HGA are different clinical entities. D2-hydroxyglutaric aciduria is a neurometabolic disorder characterized by epilepsy, hypotonia and psychomotor retardation, caused by the mutations in the $\mathrm{D} 2 \mathrm{HGDH}$ (Type 1 D2HGA) and IDH2 (Type 2 D2HGA) genes. It is important to differentiate between the different forms

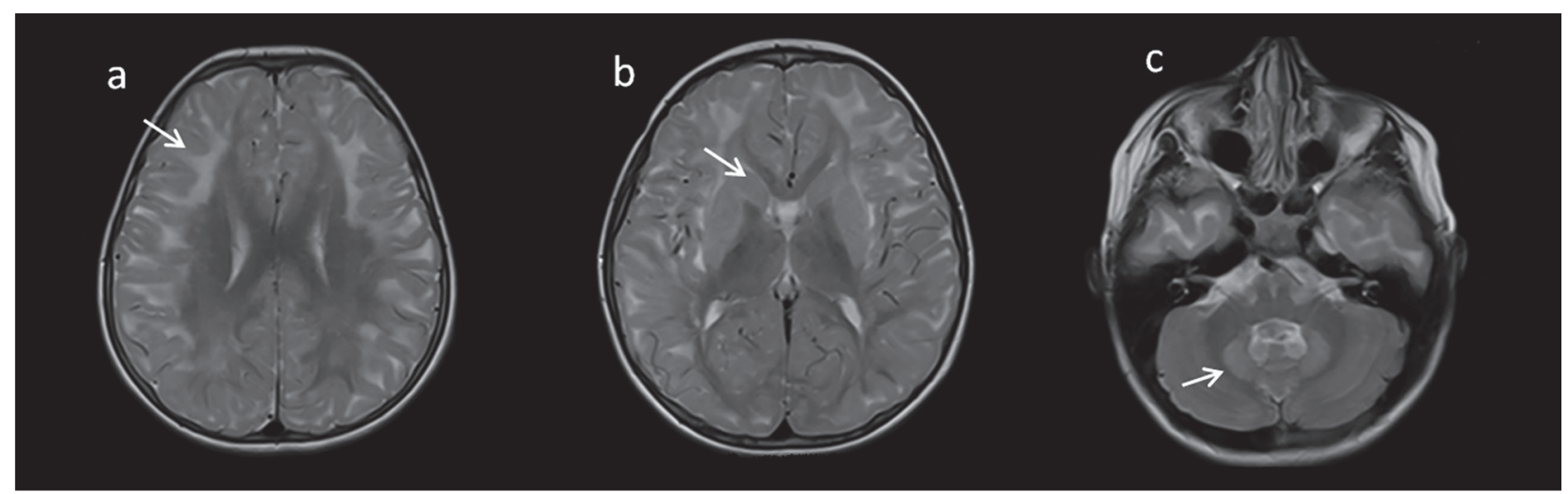

Figure 1. (a) Subcortical white matter hyperintensity on T2 weighted imaging; (b) bilateral nuclei caudatus and putamen hyperintensity on T2 weighted imaging; (c) bilateral dentate nuclei hyperintensity on T2 weighted imaging.

Tandem mass spectrometry (MS/MS) analysis using dried blood spots revealed normal results. Urine organic acid analysis peformed with gas chromatography-mass spectrometry (GC-MS) method showed increased excretion of L2HG acid $(250.87 \mathrm{mmol} / \mathrm{mol} \mathrm{cr}$, normal range: 1.3-13.9). Analysis of the $L 2 H G D H$ gene showed a novel mutation at c.368A $>$ G, p.(Tyr123Cys). The parents were shown to carry the same mutation in a heterozygous state.

Riboflavin (vitamin B2) $150 \mathrm{mg} / \mathrm{d}$ and carnitine 100 $\mathrm{gm} / \mathrm{kg} /$ day was initiated. The patient has been followed at our clinic for 4 years. No change in the amount of L2HG acid excretion has been detected since. He had a right focal seizure at the age of 10 years with no pathological findings in electroencephalography (EEG). He is now in a stable condition with no deterioration in neurological condition. Cranial MRI has been repeated recently, with no signs of progression of disease and without any findings of intra cerebral malignancies.

\section{DISCUSSION}

The differential diagnosis of L2HGA begins with the clinical evaluation of a patient with unexplained developmental delay and neurological findings of unknown etiology. Urinary organic acid screening with GC-MS, performed as a part of metabolic screening, of L2HGA in order to provide genetic counseling and future prenatal diagnosis [1].

Although the underlying mechanisms are largely unclear, in animal models, L2HG acid has been shown to impair the activities of the enzymes cytochrome $\mathrm{C}$-oxidase and ATP-synthase that play a major role in the oxidative phosphorylation. To date, there are approximately 100 documented cases in the medical literature [1-3]. Varying levels of urine L2HG levels have been previously reported (ranging from 350.0 to $3357.0 \mathrm{mmol} / \mathrm{mmol}$ creatinine). Urine L2HG of our patient was found to be similar to the cases reported in the literature [1].

Affected individuals have neurological manifestations including mild-to-moderate psychomotor retardation, loss of milestones, intention tremor, cerebellar ataxia, pyramidal and extrapyramidal symptoms and epilepsy. Macrocephaly has been reported in some cases diagnosed with L2HGA in the literature [4,5]. The disease may lead to chronic encephalopathy, without any symptoms of acute metabolic attacks. The progression of L2HGA and the consequent disability may vary between patients, which is a factor that may cause a delay in diagnosis [1]. At the time of diagnosis, the complaints of our patient were mild, including only hand tremors. Although macrocephaly and a slight ataxic gait were also present, this was not noticed at his previous visits to the pediatric clinic for routine follow-up. 
Our patient carries the homozygous c. $368 \mathrm{~A}>\mathrm{G}$, p.(Tyr123 Cys) mutation, which has not been previously reported according to the Leiden Open Variation Database (http:// www.LOVD.n1/L2HGDH2011), where numerous mutations in the $L 2 H G D H$ gene have now been reported worldwide [6]. We used the SIFT (https://sift.bii.a-star.edu. $\mathrm{sg} /$ ) and Mutationtaster (http://www.mutationsater.org/) to predict the possible impact of the amino acid substitution on the structure and function of the protein L2HGDH. The test results indicated it was a missense mutation, probably damaging and disease-causing. This mutation also showed deleterious and highly pathogenic effects on evolutionary highly conserved amino acid according to the LRT (https://dmpi.duke.eud/combined-likelihood-ratio-testcandidate-gene-studies), and MutationAssessor (http:// mutationassessor.org) databases. The parents were shown to carry the same mutation in a heterozygous state. This suggests that the mutation is probably pathogenic. Further functional tests are needed.

Cranial MRI findings are very specific and include subcortical white matter signal changes, with cerebellar cortical atrophy and signal abnormalities in the basal ganglia and dentate nuclei despite deep white matter protection [7]. Moroni et al. [8] observed a good correlation between the severity of the disease and the extent of lesions on MRI. Cerebral neoplasms may also occur [810] and rapid deterioration in L2HGA would not suggest a metabolic derangement but rather complications of a cerebral malignancy [8]. Brain tumors have been related to the metabolite $\mathrm{L} 2 \mathrm{HG}$ acid that have also been known to be an oncometabolite. Shim et al. [11] have identified elevated L2HG acid levels in renal cells affected with clear cell renal carcinoma (RCC), especially the L-enantiomer being the predominant form, and have shown L2HG acid levels to be inversely correlated with L2HGDH expression. Furthermore, they have shown $\mathrm{L} 2 \mathrm{HGDH}$ reconstitution in RCC cell lines to suppress in vitro tumor phenotypes and have suggested that L2HGDH has metabolic tumor suppressor activity [11]. As the mutation c.368A $>$ G, p.(Tyr $123 \mathrm{Cys}$ ) in the $\mathrm{L} 2 H G D H$ gene has not been previously reported, we do not have information regarding the clinical consequences of L2HGA in our patient.

Although there are no specific therapeutic approaches, supplementation of and flavin adenin dinucleotide (FAD) and riboflavin (precursor of FAD) have been reported to be effective in some patients, especially in the ones with mild mutations [1-3]. Unfortunately, these do not improve the leukoencephalopathy. Our patient's symptoms have now been stabilized. Although he had an episode of a focal seizure, his neurological complaints including hand tremors and ataxic gait have not deteriorated within 4 years follow-up.

\section{CONCLUSIONS}

L-2-hydroxyglutaric aciduria is a cerebral organic aciduria that may lead to various neurological complications. Early recognition of symptoms and initiation of supportive therapy may slow down the progression of the disease. It is necessary to keep in mind that the symptoms of various inborn errors of metabolism may be obscure and detailed neurological examination should be a part of routine pediatric visits. Cranial MRI studies may be helpful to diagnose patients who are suspected to have a neurometabolic disorder.

\section{ACKNOWLEDGMENTS}

All authors contributed to the scientific work of this study. This case has been presented as a poster at the Annual Symposium of the Society for the Study of Inborn Errors of Metabolism, Lyon, France, on September 1-4, 2015. We thank to Dr. Gajja Salomons [Centre for Genetic Diseases, VUmc (VU Medisch Centrum), Amsterdam, The Netherlands] and her laboratory for their collaboration in the molecular genetic study of our case.

Declaration of Interest. The authors report no conflicts of interest. The authors alone are responsible for the content and writing of this article.

\section{REFERENCES}

1. Kranendijk M, Struys EA, Salomons GS, Van der Knaap MS, Jakobs C. Progress in understanding 2-hydroxyglutaric acidurias. J Inherit Metab Dis. 2012; 35(4): 571-587.

2. Ma S, Sun R, Jiang B, Gao J, Deng W, Liu P, et al. L2hgdh deficiency accumulates 1-2-hydroxyglutarate with progressive leukoencephalopathy and neurodegeneration. Mol Cell Biol. 2017; 37(8). pii: e0049216. doi: 10.1128/ MCB. 00492-16.

3. Steenweg ME, Jakobs C, Errami A, van Dooren SJ, Adeva Bartolomé MT, Aerssens P, et al. An overview of L-2-hydroxyglutarate dehydrogenase gene (L2HGDH) variants: A genotype-phenotype study. Hum Mutat. 2010; 31(4): 380-390.

4. Faiyaz-Ul-Haque M, Al-Sayed MD, Faqeih E, Jamil M, Saeed A, Amoudi MS, et al. Clinical, neuroimaging, and genetic features of L-2-hydroxyglutaric aciduria in Arab kindreds. Ann Saudi Med. 2014; 34(2): 107-114.

5. Topcu M, Jobard F, Halliez S, Coskun T, Yalcinkayal C, Gerceker FO, et al. L-2-hydroxyglutaric aciduria: 
Identification of a mutant gene C14orf160, localized on chromosome 14q22.1. Hum Mol Genet. 2004; 13(22): 2803-2811.

6. Leiden Open Variation Database, 2015. http://gre nada.lumc.nl/LOVD2/vumc/home.php? select $\mathrm{db}=\mathrm{L} 2 \mathrm{HGDH}$. Accessed 17 March 2015.

7. Steenweg ME, Salomons GS, Yapici Z, Uziel G, Scalais E, Zafeiriou DI, et al. L-2-hydroxyglutaric aciduria: Pattern of MR imaging abnormalities in 56 patients. Radiology. 2009; 251(3): 856-865.

8. Moroni I, Bugiani M, D’Incerti L, Maccagnano C, Rimoldi M, Bissola L, et al. L-2-hydroxyglutaric aciduria and brain malignant tumors: A predisposing condition? Neurology. 2004; 62(10): 1882-1884.
9. Patay Z, Mills JC, Löbel U, Lambert A, Sablauer A, Ellison DW. Cerebral neoplasms in L-2 hydroxyglutaric aciduria: 3 new cases and meta-analysis of literature data. AJNR Am J Neuroradiol. 2012; 33(5): 940-943.

10. Haliloglu G, Jobard F, Oguz KK, Anlar B, Akalan $\mathrm{N}$, Coskun T, et al. L-2-hydroxyglutaric aciduria and brain tumors in children with mutations in the $\mathrm{L} 2 \mathrm{H}-$ GDH gene: Neuroimaging findings. Neuropediatrics. 2008; 39(2): 119-122.

11. Shim EH, Sudarshan S. Another small molecule in the oncometabolite mix: L-2-hydroxyglutarate in kidney cancer. Oncoscience. 2015; 2(5): 483-486. eCollection 2015. 